\title{
High grade dysplasia of the gastric mucosa: a marker for gastric carcinoma
}

\author{
M Lansdown, P Quirke, M F Dixon, A T R Axon, D Johnston
}

\begin{abstract}
The natural history of gastric epithelial dysplasia and its relation to gastric cancer are ill defined. A consecutive series of $\mathbf{4 0}$ patients with an initial diagnosis of gastric epithelial dysplasia based on examination of endoscopic biopsies has been reviewed to determine the clinical outcome and to evaluate a two tier histological grading system as a predictor of the risk of cancer. On review, only 20 of the 40 patients were considered to have true dysplasia: seven patients had low grade dysplasia and 13 had high grade dysplasia. Of the 13 patients with high grade dysplasia, $11(85 \%)$ were found to have gastric cancer within 15 months. Of the 10 patients with high grade dysplasia who underwent gastrectomy, six were found to have early gastric cancer, three had cancer invading into the muscularis propria, and none had lymph node metastases. High grade dysplasia is thus a marker of gastric cancer. Moreover, the cancers associated with high grade dysplasia are usually pathologically favourable and curable. The finding, by an experienced pathologist, of high grade dysplasia in two separate sets of endoscopic biopsies is therefore an indication for radical surgical treatment, provided that the patient's age and general condition permit such an approach.
\end{abstract}

Little is known about the natural history of epithelial dysplasia in the stomach. Areas of epithelial dysplasia can often be found adjacent to established gastric cancer..$^{1-3}$ It is tempting to propose that gastric cancer arises from dysplastic epithelium just as colonic cancer arises from adenomas. ${ }^{+}$Follow up studies of patients with dysplasia of the gastric epithelium tend to support this hypothesis, ${ }^{15-8}$ though the rate of progression to cancer appears to be slow.

A firm diagnosis of dysplasia made on the basis of endoscopic biopsies raises two main problems: (a) Has gastric cancer been missed because of sampling error? (b) If dysplasia is present, is the risk of progression to cancer so great that the patient should be advised to undergo gastrectomy?

The degree or severity of dysplasia is obviously important. Gastric epithelial dysplasia is graded by most pathologists into mild, moderate, and severe categories. Cancer is diagnosed only if neoplastic cells breach the basement membrane of the epithelium and invade the lamina propria. The use of terms such as 'intraepithelial cancer' and 'carcinoma in situ' for lesions with the cytological appearances of cancer but without evidence of invasion of the lamina propria is discouraged. ${ }^{9}$ The difficulties encountered in interpreting the changes in gastric biopsy specimens are similar to those experienced with ulcerative colitis, and therefore a system with only two grades of dysplasia has much to commend it. ${ }^{10} \mathrm{~A}$ potential advantage of such a simple two grade classification is that the natural history and prognostic importance of each category can be identified more readily, which in turn allows more precise guidelines for clinical management to be formulated.

In this study we sought to determine the fate of all patients who were diagnosed as having gastric epithelial dysplasia over a five year period and to evaluate a two tier histological grading system as a predictor of the risk of cancer.

\section{Methods}

During the five years 1983 to 1987 inclusive, gastric epithelial dysplasia was diagnosed in 40 patients at the Leeds General Infirmary, and categorised by the reporting pathologist into mild, moderate, or severe grades $^{9}$ or, more recently, into low grade or high grade dysplasia. ${ }^{10}$ All the original biopsy specimens and any subsequent pathological material were reviewed and reclassified as (1) no dysplasia, (2) low grade dysplasia, (3) high grade dysplasia, and (4) carcinoma. Gastric cancer was classified

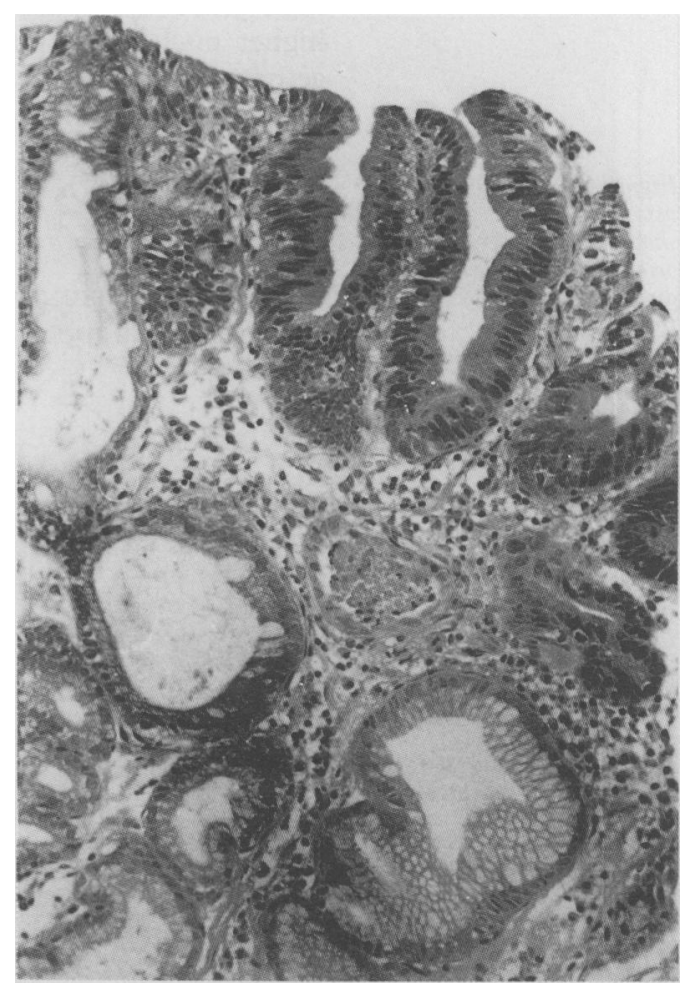

Figure 1: Biopsy specimen categorised as low grade dysplasia, showing minor stratification of elongated, hyperchromatic nuclei, extending up to the surface of the mucosa $(\times 640)$. 


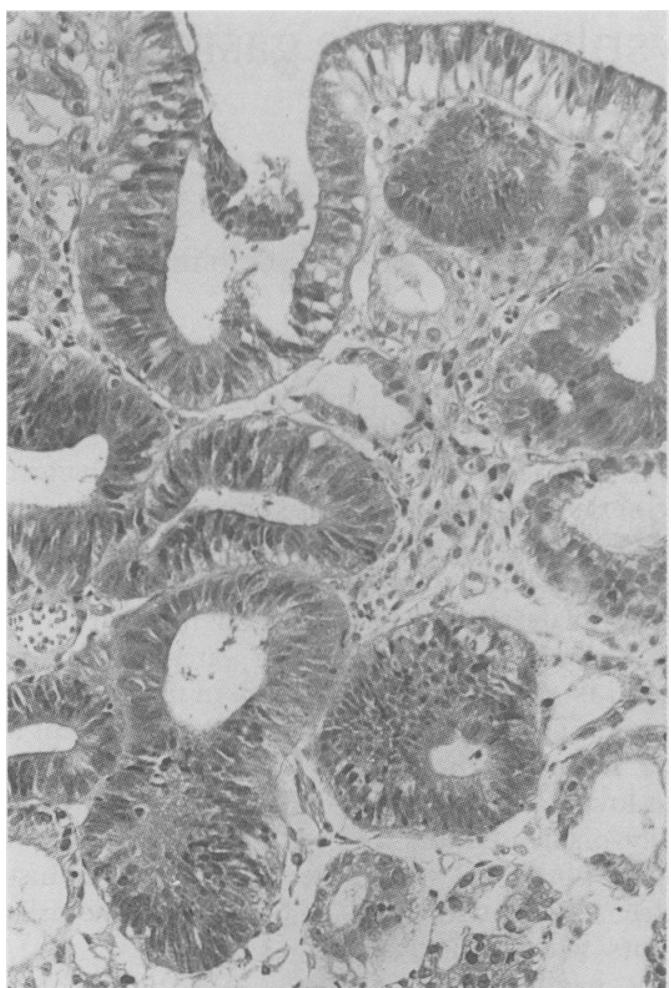

Figure 2: Biopsy specimen showing high grade dysplasia, in which the nuclei are much more pleomorphic than in Figure 1. Pseudostratification occupies the full thickness of the epithelium and there are misplaced mucin vacuoles $(\times 640)$.

according to the revised TNM classification of malignant tumours."

DIAGNOSIS OF DYSPLASIA

The cytological and architectural features of dysplasia of the gastric mucosa are well described. ${ }^{19^{12-14}}$ Diagnosis of low grade (Fig 1) or high grade dysplasia (Fig 2) depended on the higher nucleus:cytoplasm ratio, higher mitotic activity, greater loss of polarity, pseudostratification, more pronounced nuclear pleomorphism, and more prominent nucleoli in high grade

Figure 4: Campylobacterassociated chronic gastritis showing surface erosion, originally diagnosed as mild dysplasia. In this case the foveolae show simple regenerative hyperplasia $(\times 640)$.

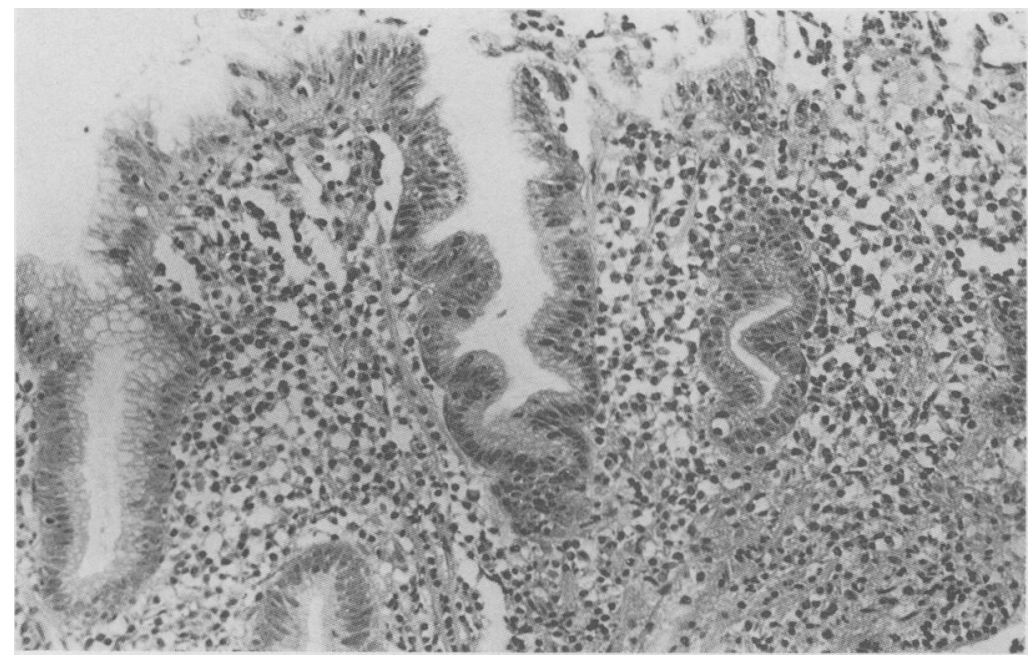

DIAGNOSIS OF CARCINOMA

The cytological appearances of neoplastic cells in

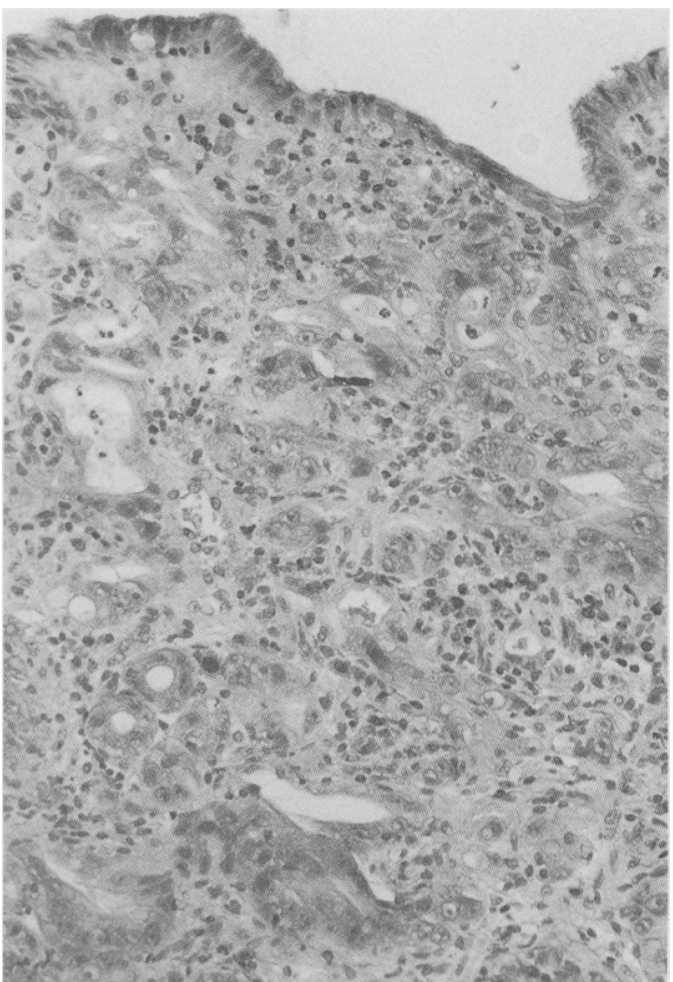

Figure 3: Biopsy specimen from a patient not included in this series where the bizarre appearances were interpreted as intramucosal carcinoma, but in whom no cancer was detected in a subsequent gastrectomy specimen. Biopsy specimens showing either frank intramucosal carcinoma or bizarre 'suspicious' appearances have not been classified as gastric dysplasia $(\times 640)$

gastric cancer and in high grade dysplasia are often identical. Carcinoma was diagnosed only if neoplastic cells were seen to be invading the lamina propria. Multiple sections through biopsy material were examined to reduce the likelihood of invasion being missed. Cases in which the original endoscopic biopsy material showed bizarre cytological and architectural appearances suspicious of carcinoma (Fig 3), such as are sometimes seen in biopsy specimens from the margins of a tumour, were excluded from the study. Biopsy material with these appearances should not be classified as dysplasia.

\section{Results}

After histological review of the original specimen seven patients were classified as having low grade and 13 as having high grade dysplasia. In 19 of the 40 cases reviewed the appearances were not considered to represent true dysplasia, and in one case the diagnosis was changed to intramucosal carcinoma. The revised diagnoses in these patients are presented in Table I. These 20 cases were originally classified as mild, moderate, or low grade dysplasia, usually by 'non-specialist' pathologists (Figs 4 and 5).

\section{PATIENTS WITH DYSPLASIA}

Clinical details of the 20 patients with confirmed gastric epithelial dysplasia were obtained from the case notes. Their median age was 72 years (range 55-86 years). Eight were women and 12 men. Five of the 20 patients had been operated on for benign peptic ulceration, by means of 
TABLE I Pathological conditions of the stomach that were initially mistaken for dysplasia

\begin{tabular}{lr}
\hline Revised diagnosis & $N o$ \\
\hline Gastric carcinoma & 1 \\
Chronic atrophic gastritis (intestinal metaplasia with & 7 \\
hyperproliferative 'crypts') & 6 \\
Chronic gastritis with erosions (regenerative atypia) & 1 \\
Chronic superficial gastritis (foveolar hyperplasia) & 4 \\
Reflux gastritis (foveolar hyperplasia) & 1 \\
Lymphocytic gastritis (erosion with regenerative atypia) & 20 \\
Total & \\
\hline
\end{tabular}

Polya gastrectomy or gastroenterostomy, 15-50 years previously (Table II).

\section{CLINICAL PRESENTATION}

There is a policy of open access to endoscopy at the General Infirmary, but there is no screening programme for asymptomatic patients. Thus all the patients in this study had undergone endoscopy because they had symptoms suggestive of upper gastrointestinal disease such as pain, loss of weight, or anaemia. The indications for endoscopy are given in Table III.

ABNORMALITIES OF THE GASTRIC MUCOSA SEEN AT ENDOSCOPY (TABLE IV)

Gastric ulcers and tumours or polyps were by far the commonest abnormalities seen at endoscopy.

\section{Gastric ulceration}

Seven of the 13 patients with high grade dysplasia were found to have gastric ulceration at the first endoscopy: one patient had two superficial ulcers on the lesser curve and six patients each had a single ulcer situated on the lesser curve. The largest ulcer was $2 \mathrm{~cm}$ in diameter. Only one of these ulcers was described as malignant by the endoscopist, though in another case the presence of irregular mucosa near the ulcer raised suspicion of early gastric cancer. Healing of the ulcer in response to treatment with $\mathrm{H}_{2}$ receptor antagonists was documented in four of these patients at the time of the second endoscopy.

Two of the seven patients with low grade dysplasia had an ulcer on the lesser curvature. In one patient the ulcer was described as an erosion: turnover that occurs in metaplastic mucosa and not to a premalignant change $(\times 64)$.

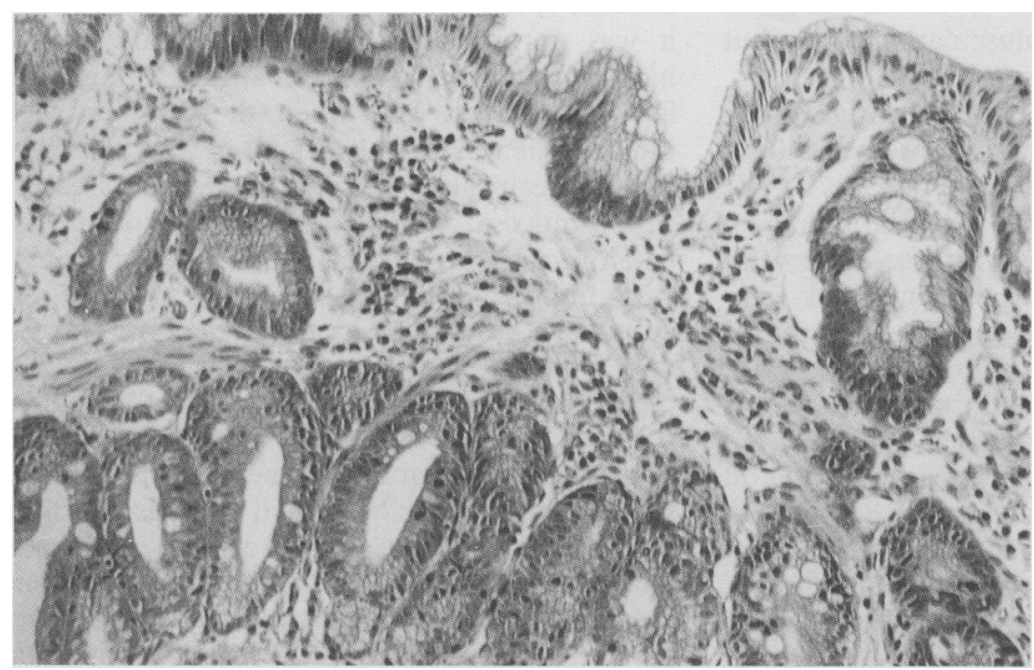

TABLE II Nature of previous operations for benign peptic ulceration in patients found to have dysplasia in endoscopic biopsies of the stomach

\begin{tabular}{llll}
\hline No & Operative procedure & $\begin{array}{l}\text { Degree of } \\
\text { dysplasia }\end{array}$ & $\begin{array}{l}\text { Years between } \\
\text { operation and } \\
\text { diagnosis of dysplasia }\end{array}$ \\
\hline 1 & Polya partial gastrectomy & High grade & 15 \\
2 & Polya partial gastrectomy & High grade & 31 \\
3 & Gastroenterostomy & High grade & 50 \\
4 & $\begin{array}{l}\text { Polya partial gastrectomy } \\
\text { Low grade }\end{array}$ & 30 \\
5 & $\begin{array}{c}\text { Truncal vagotomy and } \\
\text { gastroenterostomy }\end{array}$ & Low grade & 21 \\
\hline
\end{tabular}

unfortunately this patient did not return for follow up. The other patient had an ulcer which appeared to be benign and which healed in response to treatment with $\mathrm{H}_{2}$ receptor antagonists. Follow up biopsies six months later showed continuing low grade dysplasia in the ulcer scar.

\section{Tumour}

Two patients with high grade dysplasia had raised tumours. In one patient a tumour on the lesser curve was associated with liver metastases on the ultrasound scan. In the other patient the tumour was seen to occupy most of the antrum: no further specimens were taken and the patient underwent a total gastrectomy with radical lymphadenectomy. The resected specimen contained a tumour $8 \mathrm{~cm}$ in diameter invading the muscularis propria, but the lymph nodes were not involved.

\section{Polyp}

Three patients had high grade dysplasia in solitary polyps. One was an adenoma with a focus of high grade dysplasia, situated adjacent to a gastroenterostomy: carcinoma was found in a second set of biopsy specimens taken a week later. The resected specimen contained an adenoma $3 \mathrm{~cm}$ in diameter in which intramucosal carcinoma was found. One patient with a small sessile polyp high on the posterior wall of the stomach was found to have carcinoma at the same site in a second set of specimens taken five months later. The third patient had high grade dysplasia in a sessile antral polyp which at endoscopy was thought to measure $1 \mathrm{~cm}$ in diameter: further sets of biopsy specimens taken two and three months later showed continuing high grade dysplasia.

One patient had multiple tiny regenerative polyps situated on the posterior wall of the upper part of the stomach. Endoscopic biopsy specimens from this region showed changes of intestinal metaplasia and regeneration and also low grade dysplasia. Further specimens taken 14 months later also showed low grade dysplasia,

TABLE III Indications for endoscopy in 20 patients with gastric epithelial dysplasia

\begin{tabular}{ll}
\hline Indication for endoscopy & No of patients \\
\hline Upper abdominal pain & $15^{\star}$ \\
Anaemia & 4 \\
Melaena & 1 \\
\hline
\end{tabular}

^Four patients who presented with upper abdominal pain also complained of loss of weight. 
TABLE IV Findings at endoscopy in 20 patients with gastric epithelial dysplasia

\begin{tabular}{lll}
\hline $\begin{array}{l}\text { Abnormality seen } \\
\text { at endoscopy }\end{array}$ & $\begin{array}{l}\text { Low grade } \\
\text { dysplasia }\end{array}$ & $\begin{array}{l}\text { High grade } \\
\text { dysplasia }\end{array}$ \\
\hline Ulcer & 2 & 7 \\
Raised tumour or polyp & 1 & 5 \\
Plaque & 0 & 1 \\
Other (see text) & 4 & 0 \\
\hline
\end{tabular}

but a third set taken after a further seven months showed only chronic atrophic gastritis and intestinal metaplasia.

\section{Plaque}

One patient with high grade dysplasia had a plaque on the lesser curve. An ulcerated early gastric cancer was suspected by the endoscopist. Two further sets of biopsy specimens confirmed the presence of high grade dysplasia before the patient underwent gastrectomy. The entire area of dysplasia was examined histologically; the 'ulcer' was found to be a depressed area of epithelium. Since no evidence of invasion of the lamina propria was found the lesion was not classified as a carcinoma.

\section{Other abnormalities}

The endoscopic abnormality seen in the remaining four'patients, all of whom had low grade dysplasia, was 'atrophic mucosa' in two, irregular mucosa at a gastroenterostomy in one, and deformity of the pyloric canal in one.

\section{CLINICAL COURSE}

\section{Patients with high grade dysplasia}

One patient was thought to have gastric cancer at endoscopy and liver metastases were found by ultrasound scanning. One set of biopsy specimens was taken which showed only high grade dysplasia. Since he was unfit for surgical treatment, no further biopsies were carried out.

One patient with a large antral tumour, biopsy specimens of which showed high grade dysplasia, underwent a total gastrectomy with radical lymphadenectomy, without undergoing further endoscopy. The resected stomach contained an adenocarcinoma $8 \mathrm{~cm}$ in diameter which was invading the muscularis propria but not involving lymph nodes.

The remaining 11 patients with high grade dysplasia all had at least one further set of biopsy specimens taken at a repeat endoscopy. Four patients were found to have cancer in follow up biopsies within eight months; three underwent radical gastrectomy, of whom two had early gastric cancer ( $\mathrm{T} 1 \mathrm{NO} \mathrm{M} 0$ ) and one had cancer invading the muscularis propria (T2 N0 M0). The fourth patient, who had cancer in a polyp, was unfit for surgical treatment and was referred for laser photocoagulation: repeat biopsies at first showed no further evidence of cancer, but one year after laser treatment biopsy specimens showed the presence of recurrent carcinoma.

In seven patients follow up endoscopy and biopsy over 1-15 months showed continuing high grade dysplasia without evidence of cancer. Six of these seven patients underwent radical gastrectomy, and five of them were found to have cancer: four had early gastric cancer (T1 N0 M0) and one had a T2 N0 M0 tumour. In the sixth patient, examination of the gastrectomy specimen showed high grade dysplasia but there was no evidence of carcinoma (the entire dysplastic area was processed for microscopic examination). The remaining patient with continuing high grade dysplasia was referred for laser photocoagulation of a small antral polyp. After destruction of the polyp there was regression to low grade dysplasia and then no dysplasia was found in three subsequent sets of specimens taken over a period of one year. Eighteen months after photocoagulation, however, a raised lesion was again seen at endoscopy: biopsy specimens showed no evidence of dysplasia, but because of the high degree of suspicion a further set was taken and the presence of high grade dysplasia was confirmed. This patient is a poor operative risk and is returning for further biopsies before a decision about further treatment is made.

The TNM staging of the 13 patients with high grade dysplasia in the original set of biopsy specimens is summarised in Table V.

\section{Patients with low grade dysplasia}

Of the seven patients with low grade dysplasia, four underwent further endoscopy and biopsy and three did not. In the four patients further biopsies after 2-24 months showed no evidence of dysplasia in three, while there was continuing low grade dysplasia in one patient after six months. Of the three patients who were not followed up, one failed to attend for further investigation, one was discharged, and the third died of an unrelated condition.

\section{Patients reclassified as not having dysplasia}

In 19 patients the diagnosis was revised to gastritis with regenerative atypia (Table I). None of these patients developed gastric carcinoma during a median follow up period of 18 months.

\section{NUMBER OF BIOPSY SPECIMENS}

Because of the retrospective nature of this review it was not possible to determine how many biopsy specimens were taken at each endoscopy in each patient, and when the number was indicated it was not always clear whether they had all been taken from the abnormal area of gastric mucosa. The number of sets of specimens taken in each patient was recorded. In patients with high grade dysplasia in the first set of specimens, in whom gastric cancer was diagnosed on the basis of follow up biopsies, the cancer was always found in either the second or the third set of specimens. Only one patient underwent gastrectomy on the basis of a single set of specimens that showed high grade dysplasia: this was because he had a large, malignant looking antral tumour at endoscopy. The other six patients who underwent gastrectomy on account of high grade dysplasia had two to five sets 
TABLE V Endoscopic appearance, treatment, TNM stage, and interval between diagnosis of high grade dysplasia and gastric cancer in 13 patients who were initially found to have high grade dysplasia present in endoscopic biopsies

\begin{tabular}{rllllr}
\hline Patient & $\begin{array}{l}\text { Endoscopic } \\
\text { appearance }\end{array}$ & Treatment & TNM stage & $\begin{array}{l}\text { Interval between diagnosis } \\
\text { of high grade dysplasia and } \\
\text { diagnosis of gastric cancer } \\
\text { (months) }\end{array}$ \\
\hline 1 & Plaque & Subtotal gastrectomy & T0 N0 M0 & - \\
2 & Polyp & Total gastrectomy & T1 N0 M0 & $<1$ \\
3 & Ulcer & Subtotal gastrectomy & T1 N0 M0 & 2 \\
4 & Ulcer & Subtotal gastrectomy & T1 N0 M0 & 3 \\
5 & Ulcer & Total gastrectomy & T1 N0 M0 & 5 \\
6 & Ulcer & Total gastrectomy & T1 N0 M0 & 6 \\
7 & Ulcer & Total gastrectomy & T1 N0 M0 & 13 \\
8 & Raised tumour & Subtotal gastrectomy & T2 N0 M0 & 2 \\
9 & Ulcer & Subtotal gastrectomy & T2 N0 M0 & 8 \\
10 & Ulcer & Subtotal gastrectomy & T2 N0 M0 & 15 \\
11 & Polyp & Laser photocoagulation & Tx Nx Mx & - \\
12 & Polyp & Laser photocoagulation & Tx Nx Mx & 5 \\
13 & Raised tumour & No treatment & Tx Nx M1 & - \\
\hline
\end{tabular}

Note: Cancer was only diagnosed in patients $3,4,5,7,8$, and 9 after examination of the resected stomach. Patient 13 was terminally ill with disseminated cancer at the time of diagnosis. Patients 11 and 12 did not undergo gastrectomy: patient 11 had at least high grade dysplasia, and patient 12 had at least a $\mathrm{Tl}$ cancer. resected for advanced cancer, areas of epithelial dysplasia can also be found. ${ }^{215}$ This strong association between gastric cancer and synchronous dysplasia should thus arouse strong suspicion that gastric cancer has been missed when biopsy specimens show the presence only of high grade dysplasia.

A problem of management arises when repeated endoscopies and biopsies confirm the presence of high grade dysplasia, but not of frank carcinoma. Some of the patients in the present study may have had a small area of invasive cancer, within an area of high grade dysplasia, which was missed by repeated biopsies, as borne out by the finding of invasive cancer in six of the seven patients who underwent gastrectomy but who did not have cancer in the preoperative endoscopic biopsy specimens. Admittedly, two of these patients had high grade dysplasia diagnosed more than a year before they underwent gastrectomy, and they may have developed invasive cancer during that period of follow up. Cancer detected soon after dysplasia is diagnosed probably represents synchronous disease, but cancer found after many months of follow up, with repeated biopsies, may represent true progression. Gastric cancer has been found in patients with dysplasia who were followed up for periods of up to six years..$^{6-8}$

The finding of occult cancer in patients with gastric epithelial dysplasia who underwent gastrectomy has been described previously. Thus, Aste $e t a l^{16}$ found gastric cancer in eight of 13 patients with moderate or severe dysplasia who underwent gastrectomy. As in the present study, dysplasia was usually associated with a visible lesion, most commonly an ulcer, and many of the cancers detected were 'early' and potentially curable. Similarly, Saraga $e t a l^{6}$ found cancer in 17 of 21 patients with severe dysplasia within three years of the original diagnosis of severe dysplasia. In 12 of these 17 patients the carcinoma was only found on examination of the gastrectomy specimen, and in eight patients it was early gastric cancer. Previous reports of gastric cancer developing in areas of dysplasia may have underestimated the true incidence of gastric cancer if the whole area of dysplasia was not subjected to histological examination.

Attempts to try to distinguish three different grades of severity of dysplasia may encourage the use of the term 'mild dysplasia' when the pathologist is presented with any biopsy specimen that shows minor cytological atypia. Many of these cases will represent regenerative atypia rather than a premalignant change and the patient will be subjected to unnecessary surveillance and anxiety. Of those patients with 'true' dysplasia the pathologist needs to identify a low risk group of patients who can safely be followed up by means of endoscopy and biopsy, and a high risk group of patients in whom the risk of cancer is sufficiently high to justify the use of gastrectomy. A group of patients with dysplasia of 'intermediate' severity is likely to contain patients of low and high risk, and inclusion of such a category therefore impedes clinical decision making. A further difficulty is that biopsy specimens taken at endoscopy are small and their interpretation may be difficult. ${ }^{17}$ In the
It seems likely that in some patients the presence of carcinoma may have been missed in the original set of biopsy specimens, which showed only an adjacent area of high grade dysplasia. In $20-40 \%$ of stomachs resected for early gastric cancer, and up to $80 \%$ of stomachs 
present study, when biopsy material was reviewed it was found that 16 of 23 cases originally reported as mild or low grade dysplasia had been misdiagnosed. Fifteen were reclassified as showing gastritis, with regenerative atypia, but one was reclassified as frank carcinoma. In contrast, no set of specimens originally thought to show severe or high grade dysplasia was reclassified as gastritis alone. Of the eight sets of specimens originally classified as moderate dysplasia, five were reclassified as gastritis, two as high grade dysplasia, and one as low grade dysplasia. No patient whose biopsy specimens were reclassified as gastritis developed gastric carcinoma during a median period of follow up of 18 months.

In this study dysplasia was most commonly associated with an ulcer. This is in keeping with the findings of previous studies. ${ }^{616}$ The finding of dysplasia in the margin of a peptic ulcer would suggest that the ulcer is either malignant or about to become malignant, but the risk of cancer developing in a benign ulcer is small ${ }^{1}$ and many of the putative dysplastic changes at the edges of ulcers are probably regenerative and not neoplastic. Problems in the diagnosis of dysplasia and cancer in biopsy material taken from the edge of an ulcer have been highlighted by Williams. ${ }^{17}$ The problem is particularly acute in the presence of erosions where regenerative activity in residual glands of the deep mucosa can be readily mistaken for dysplasia or even carcinoma. ${ }^{17}$ 'Overdiagnosis' of dysplasia in some series may explain why so few patients with dysplasia were subsequently found to have cancer.

It may also be the case that neoplastic and dysplastic epithelium is prone to ulceration, and some ulcers initially thought to be benign are in fact neoplastic. Farinati et $a l^{7}$ discovered 10 cancers among 144 patients with apparently benign ulcers who were followed up for 41 months with repeated endoscopy and biopsy. Most cancers were found within six months, so it is likely that the original biopsy specimens were either not representative of the ulcer or were misinterpreted.

The difficulty in distinguishing benign from malignant ulceration in the stomach by endoscopic examination is well known. This is particularly so for early gastric cancer, as shown by Ballantyne $e t a l^{19}$ in whose study of 14 ulcerating early gastric cancers, seven were thought to be benign by naked eye inspection at gastroscopy. In Japan, however, where gastric cancer is found in its early stages more often than in the West, malignant ulcers can be distinguished from benign ulcers with an accuracy rate of $80 \%$ by the endoscopist.' It was interesting that in the present study four ulcers associated with high grade dysplasia healed in response to $\mathrm{H}_{2}$ receptor antagonists. In each case the patient was subsequently found to have gastric cancer.

Polyps of the adenomatous type are rare in the stomach, comprising $10 \%$ or less of all gastric polyps. Estimates of the proportion that progress to gastric cancer vary from $6 \%$ to $75 \% .{ }^{20}$ Nakamura and Nakano ${ }^{21}$ found foci of carcinoma in $33 \%$ of those gastric polyps that were similar in histological appearance to colonic adenomas.
Probably only $5 \%$ of all gastric cancers arise in polyps. ${ }^{22}$ In the present series cancer was found in follow up biopsy material from an adenomatous polyp in one patient.

We have performed radical gastrectomies in patients who had high grade dysplasia but no histological evidence of invasive carcinoma in preoperative biopsy material. It is likely that our cases of high grade dysplasia correspond to Nagayo's category of 'probable carcinoma,' for which he advises that gastrectomy be performed. In Nagayo's vast experience, 'probable carcinomas' and 'borderline lesions' comprise less than $3 \%$ of all biopsy specimens examined. The use of gastrectomy in these patients, however, presumably increased the number of early gastric cancers resected and may have contributed to the impressive statistics for survival after surgery for gastric cancer in Japan.

In high risk patients less radical measures than aggressive surgical treatment may have to be considered. Thus two patients in our series, who were considered unfit for gastrectomy, underwent laser photocoagulation, one for carcinoma and the other for high grade dysplasia. Although the lesions recurred in both patients after 12 to 18 months' follow up, we believe that the use of laser photocoagulation is a better option than simple observation and repeated biopsy. Laser photocoagulation has also been used by Takemoto ${ }^{23}$ to treat patients with early gastric cancer who were considered unfit for gastrectomy. Endoluminal ultrasound was used to determine the depth of invasion of the cancer before treatment, and to assess the depth of destruction caused by the laser. Among 14 patients in whom the laser was thought to have coagulated the carcinoma completely, no evidence of recurrent cancer was found in 12 patients six to 22 months later, and two patients had recurrent cancer. Thus the use of endoluminal ultrasound for the assessment and follow up of early gastric cancer treated by laser photocoagulation merits further study in selected elderly or unfit patients.

Our series shows that a high proportion (85\%) of patients with high grade dysplasia in endoscopic biopsy specimens have (or soon will have) gastric cancer. A high proportion (90\%) of these cancers are at an early stage of invasion ( $\mathrm{T} 1$ or T2). Our finding that cancer was invading the muscularis propria in three of 10 patients who underwent gastric resection highlights the risk of inaction - that is, of merely following up patients with high grade dysplasia by repeated endoscopy and biopsy.

The present study has highlighted the difficulty that can be experienced in demonstrating gastric cancer in patients presenting with high grade dysplasia even when carcinoma has invaded the muscularis propria. Although none of the patients in this series had metastases to lymph nodes, the risk of lymph node metastases is directly related to the depth of invasion of gastric cancer, being $2-4 \%$ for cancer invading the mucosa, $20 \%$ for cancer invading the submucosa, and over $40 \%$ for cancer invading the muscularis propria. ${ }^{124}$ In our own series of 27 patients who had early gastric cancers resected in the last 15 years, three (11\%) had metastases to lymph nodes. We therefore consider radical 
gastrectomy with removal of the first and second tiers of lymph nodes to be the appropriate operative procedure for most patients with high grade dysplasia, but the age and general condition of the patient must obviously be taken into account and in some instances a less radical operation will be appropriate. Whether the gastrectomy should be total or subtotal depends on the site and extent of dysplasia in the stomach. In our series two patients had more than one focus of cancer, but in both cases the cancerous foci were adjacent to each other. Thus we found no evidence of widespread high grade dysplasia in the stomach, and our data lend no support to the idea that all patients with high grade dysplasia should undergo total gastrectomy. If subtotal gastrectomy is to be carried out, however, it is important that biopsy specimens be taken from all regions of the stomach before operation so that areas of abnormal mucosa may be mapped out accurately.

The conclusion from this study, that a histological diagnosis of high grade dysplasia is usually an indication for gastrectomy because of the almost invariable association of high grade dysplasia with invasive carcinoma, places a heavy responsibility on the reporting pathologist. The recognition of dysplasia, and its distinction from atypical hyperplasia consequent upon regeneration or inflammatory activity, is far from straightforward. Indeed, such changes may be so bizarre as to give rise to appearances indistinguishable from malignancy. It is obligatory therefore for the diagnosis of high grade dysplasia to be confirmed by a second set of biopsy specimens, while the biopsy specimens themselves must be reported on by very experienced pathologists. Where expert advice is not available locally, submission of biopsy specimens showing dysplasia to a specialised pathological panel for review should improve diagnostic accuracy and reduce the risk of unnecessary gastrectomy.

1 Nagayo T. Histogenesis and precursors of human gastric cancer. Research and practice. Berlin: Springer-Verlag, 1988
2 Meister $\mathrm{H}$, Holubarsch $\mathrm{Ch}$, Haferkamp O, Schlag P, Herfarth $\mathrm{Ch}$. Significance and location of atrophic gastritis and of glandular dysplasia in benign and malignant gastric disease. In: Herfarth $\mathrm{Ch}$, Schlag $\mathrm{P}$, eds. Gastric cancer. Berlin Springer-Verlag, 1979: 105-7.

3 Grundmann E, Schlake W. Histology of possible precancerous stages in the stomach. In: Herfarth $\mathrm{Ch}$, Schlag $\mathrm{P}$, eds Gastric cancer. Berlin: Springer-Verlag, 1979: 72-82.

4 Enterline HT, Evans GW, Mercado-Lugo R, Miller L, Fitts WT. Malignant potential of adenomas of colon and rectum. 7AMA 1962; 179: 322-30.

5 Offerhaus GJA, Stadt J, Huibregtse K, Tytgat GNJ. Endoscopic screening for malignancy in the gastric remnant: the clinical significance of dysplasia in gastric mucosa. $f$ Clin Pathol 1984; 37: 748-54.

6 Saraga E, Gardiol D, Costa J. Gastric dysplasia. A histological follow-up study. Am $\mathcal{F}$ Surg Pathol 1987; 11: 788-96.

7 Farinati F, Cardin F, Di Mario F, et al. Early and advanced gastric cancer during follow-up of apparently benign gastric ulcer: significance of the presence of epithelial dysplasia. f Surg Oncol 1987; 36: 263-7.

8 Cai-pu X, Wei-wen L. Follow-up observations on chronic gastritis, intestinal metaplasia and dysplasia. Chin Med $f$ (Engl) 1985; 98: 347-8.

9 Morson BC, Sobin LH, Grundmann E, Johansen A, Nagayo $T$, Serck-Hanssen A. Precancerous conditions and epithelia dysplasia in the stomach. $\mathcal{F}$ Clin Pathol 1980; 33: 711-21.

10 Riddell RH, Goldman H, Ransohoff DF, et al. Dysplasia in inflammatory bowel disease: standardized classification with inflammatory bowel disease: standardized classification with
provisional clinical applications. Hum Pathol 1983; 14: 93168 .

11 Hermanek P, Sobin LH. TNM classification of malignant tumours. Berlin: Springer-Verlag, 1987

12 Cuello C, Correa P, Zamara G, Lopez J, Murray J, Gordillo G Histopathology of gastric dysplasias: correlations with gastric juice chemistry. Am $\mathcal{F}$ Surg Pathol 1979; 3: 491-500.

13 Jass JR. A classification of gastric dysplasia. Histopatholog 1983; 7: 181-93.

14 Ming S, Bajtai A, Correa P, et al. Gastric dysplasia. Signific ance and pathologic criteria. Cancer 1984; 54: 1794-801.

15 Nagayo T. Gastric cancer preceded by severe dysplasia. Histol Histopathol 1986; 1: 171-80.

16 Aste H, Sciallero S, Pugliese V, Gennaro M. The clinical significance of gastric epithelial dysplasia. Endoscopy 1986; 18: $174-6$.

17 Williams GT. Early gastric cancer. In: Filipe MI, Jass JR, eds. Gastric carcinoma. Edinburgh: Churchill Livingstone, 1986: 173-96

18 Isaacson P. Biopsy appearances easily mistaken for malignancy in gastrointestinal endoscopy. Histopathology 1982; 6: 37789.

19 Ballantyne KC, Morris DL, Jones JA, Gregson RH, Hardcastle JD. Accuracy of identification of early gastric cancer. Br f Surg 1987; 74: 618-9.

20 Sipponen P, Kekki M, Siurala M. In: Filipe MI, Jass JR, eds. Gastric carcinoma. Edinburgh: Churchill Livingstone, 1986 : 153-71.

21 Nakamura T, Nakano G. Histopathological classification and malignant change in gastric polyps. F Clin Pathol 1985; 38: $754-64$.

22 Hermanek P. Gastric polyps and gastric cancer. In: Herfarth Ch, Schlag P, eds. Gastric cancer. Berlin: Springer-Verlag, 1979: 147-8.

23 Takemoto $T$. Laser therapy of early gastric carcinoma. Endoscopy 1986; 18 (suppl 1): 32-6.

24 Okamura T, Tsujitani S, Korenaga D, et al. Lymphadenectomy for cure in patients with early gastric cancer and lymph node metastasis. Am $\mathcal{J}$ Surg 1988; 155: 476-80. 